\title{
Peripartum cardiomyopathy: the need for a national database
}

\author{
S. I. Lok • J. H. Kirkels • C. Klöpping • \\ P. A. F. Doevendans $\cdot$ N. de Jonge
}

Published online: 27 January 2011

(C) The Author(s) 2011. This article is published with open access at Springerlink.com

\begin{abstract}
Peripartum cardiomyopathy (PPCM) is a rare and life-threatening disease that affects young women in the last month of pregnancy or within 5 months of delivery. It is a form of dilated cardiomyopathy with left-sided systolic dysfunction. The incidence rate in the Western world is estimated to be 1:3000. Symptoms of PPCM vary greatly and may be obscured by common physiological aspects of pregnancy. Therefore, the incidence rate might be higher. Echocardiography or MRI can confirm or rule out PPCM. Unfortunately, there is no specific risk factor profile available. The clinical course varies from complete recovery to deterioration of cardiac function. Patients with PPCM, especially those whose ventricular function has not returned to normal, are advised against further pregnancy. Recently, more disease-specific therapeutic strategies have been developed with promising results for prolactin blockade by bromocriptine. Increasing awareness for PPCM among general practitioners, gynaecologists and cardiologists may help to diagnose patients efficiently in order to start adequate treatment. A national registry is warranted to identify risk factor profiles and to optimise treatment strategies.
\end{abstract}

Keywords Peripartum · Cardiomyopathy · Database . Bromocriptine

\section{Introduction}

Peripartum cardiomyopathy (PPCM) is a rare and lifethreatening form of dilated cardiomyopathy with left

S. I. Lok $(\bowtie) \cdot$ J. H. Kirkels $\cdot$ C. Klöpping $\cdot$

P. A. F. Doevendans $\cdot$ N. de Jonge

University Medical Center Utrecht,

Heidelberglaan 100 ,

3584 CX Utrecht, the Netherlands

e-mail: s.lok@umcutrecht.nl ventricular systolic dysfunction that affects women in late pregnancy or in the early puerperium. The incidence of PPCM is not known. Patients with PPCM, especially those whose ventricular function has not normalised, are advised against future pregnancies.

Recently, case reports of young patients with PPCM have been published in the Netherlands Heart Journal [1, 2]. This review provides an overview of the clinical manifestation, management, risk factors and potential pathophysiological mechanisms of PPCM and emphasises the need for a national database.

\section{Definition}

Peripartum cardiomyopathy was first described in 1849 by Richie and Virchow but was not recognised as a distinct clinical entity until 1937 [3]. It is a form of dilated cardiomyopathy with left ventricular systolic dysfunction which may lead to symptoms and signs of heart failure. Based on a report from the National Heart, Lung and Blood Institute, PPCM is defined by the following four criteria [4]:

1. Development of cardiac failure in the last month of pregnancy or within 5 months of delivery;

2. Absence of an identifiable cause of cardiac failure;

3. Absence of recognisable heart disease prior to the last month of pregnancy;

4. Left ventricular systolic dysfunction demonstrated by echocardiographic criteria, such as left ventricular ejection fraction.

These criteria limit the diagnosis to the last gestational month and the first 5 months after delivery and are based on data obtained in a relatively small group of women diagnosed in one centre in an earlier diagnostic era. Later reports describe patients who presented with cardiomyop- 
athy earlier during pregnancy $[5,6]$. In addition, based on our own observations, some patients present with clear PPCM symptoms earlier than the last gestational month. Therefore, modification of the first criterion might be necessary. In addition to these criteria, it is recommended that other conditions, which may be associated with heart failure in the puerperium, are excluded before the diagnosis of PPCM is considered. These other conditions include infectious, toxic or metabolic disorders, underlying valvular or ischaemic heart disease. However, in clinical practice, it remains difficult to distinguish patients with pre-existing (asymptomatic) cardiomyopathy, progressing during pregnancy and labour, from actual PPCM patients.

\section{Epidemiology}

The real incidence of PPCM is not known. The incidence varies among geographical regions. An accepted incidence is 1 in 3000 to 1 in 4000 live births in the United States, 1 in 1000 in South Africa and 1 case per 6000 live births in Japan [7, 8]. In Haiti, the incidence has been reported to be as high as 1 in 400 live births and in parts of Nigeria 1 in 100 [9]. As the incidence in the Netherlands is not known, we have the intention to establish a national database in order to gain more insight into the epidemiology.

\section{Risk Factors}

Several risk factors have been proposed for the development of PPCM. They are too widely distributed in the maternal population to allow for screening. PPCM can occur at any age but generally occurs in women over the age of 30 years. It is thought to be more prevalent in multiparous women, pregnancies with multiple foetuses, women of African descent and in women receiving longterm tocolytic therapy (with $\beta$-adrenergic agonists). Other possible risk factors include toxaemia of pregnancy, maternal cocaine abuse and nutritional deficiencies. Finally, a history of pre-eclampsia, eclampsia or gestational hypertension is also thought to be associated with PPCM [9].

\section{Pathogenesis}

The pathogenetic mechanisms of PPCM have been difficult to study as their incidence and prevalence are too low in Western countries to allow meaningful evaluations. Therefore, the pathogenesis is poorly understood. PPCM probably develops due to a complex interaction of inflammation, haemodynamic stress during pregnancy and genetic factors. A number of contributing factors are elucidated in more detail.

\section{Inflammatory Disease}

A number of investigators have suggested myocarditis as a cause of PPCM, although the presence of documented myocarditis varies considerably within studies. This is partly related to the timing of the endomyocardial biopsy. The incidence of inflammation is higher in patients biopsied earlier after initial presentation. Furthermore, the criteria applied in diagnosing myocarditis are not congruent between studies [10-12]. The percentages of virus detected in endomyocardial biopsies of PPCM patients varied from $10 \%$ to $70 \%$, with a similar incidence in controls, suggesting no specific role for viral infection in the aetiology of PPCM [10-12]. It is worth noting that Bultmann reported that the presence of viral genomes in endomyocardial biopsies was associated with inflammatory cardiomyopathies exclusively in PPCM patients but not in control subjects [10]. Experimental data in mouse studies suggested that encephalomyocarditis viral infection increases the severity of myocardial damage in postpartum mice in comparison with nonpregnant control mice. Primary viral infection, usually in childhood, can lead to a latent lifelong viral persistence, which is of no pathogenetic significance. During pregnancy, the immune defence is impaired. As a consequence, the virus can be reactivated, leading to more severe viral infections [10].

\section{Abnormal Immune Response}

A maternal immunological response to a foetal antigen has been proposed as another potential aetiology of PPCM. During pregnancy, foetal cells cross the placenta into the maternal circulation. The foetal cells are not destroyed because of the depressed immunological state in the mother during pregnancy and due to the weak immunogenicity of the paternal haplotype of the chimeric cells [13]. If these cells lodge in the cardiac tissue, they can trigger a pathological autoimmune response. This immune response can be exacerbated by previous exposure to paternal antigens through prior pregnancies. PPCM is indeed associated with high titres of autoantibodies against certain cardiac tissue proteins [13-15]. As in other forms of heart failure, inflammatory cytokine levels are significantly elevated in patients who died of PPCM compared with survivors [15]. At present, it is unclear whether the autoimmune response plays a causal role in the development and progression of PPCM or whether it should be considered as a consequence of the disease. 


\section{Haemodynamic Stress}

During pregnancy, labour and delivery, many haemodynamic changes occur. These alterations begin during the first 5 to 8 weeks of pregnancy and reach their peak in the late second trimester. The haemodynamic alterations during pregnancy include an increase in blood volume and cardiac output, while systemic vascular resistance decreases. During labour and delivery, haemodynamic fluctuations can be profound. Stroke volume increases, and as a consequence, cardiac output will increase. Blood loss and anaemia during delivery can further compromise the haemodynamic state. The haemodynamic changes during the postpartum state are mainly due to uterine contractions and relief of caval vein compression. The cardiovascular changes return to baseline within 3 to 4 weeks following delivery but may not resolve completely until week 12 after delivery. Following pregnancy, there is a physiologically rapid reversal of cardiac hypertrophy, possibly related to preload reduction [16].

\section{Apoptosis}

Myocyte apoptosis has been thought to be a critical component in the pathological mechanisms driving PPCM and has been investigated in mice studies. Apoptotic loss of myocardial cells itself can increase haemodynamic stress through ventricular dilation and wall thinning. Therefore, it seems to play an important role in the reduction of cardiac function and leads ultimately to heart failure. A reduction of apoptosis in mice leads to less cardiac dysfunction and reduces mortality. However, complete recovery of cardiac function has been observed in PPCM patients. Therefore, the role of cardiomyocyte loss as a general key factor of PPCM pathophysiology seems unlikely, since regeneration of myocardial cells is limited [17].

\section{Genetic Factors}

There may be a genetic basis for the development of PPCM. The development of PPCM in two sisters, which was published in a recent case report, suggests that women with a positive family history of PPCM may have an increased risk for the development of this disease [18]. Furthermore, a current investigation in the Netherlands suggests that a subset of PPCM is an initial manifestation of familial dilated cardiomyopathy. Therefore, presymptomatic screening is suggested for covert dilated cardiomyopathy in first-degree family members of PPCM patients who do not show full recovery of cardiac function. In addition, extensive monitoring in the puerpe- rium of first-degree family members of patients with PPCM is advised [19].

\section{Cardioprotective Pathways During Pregnancy}

Pregnancy is a physiological state associated with enhanced oxidative stress related to high metabolic turnover and elevated tissue oxygen requirements. During pregnancy, the heart develops a reversible physiological enlargement in response to mechanical stress and increased cardiac output. In order to protect the heart, activation of the STAT3 pathway is necessary. STAT3 promotes myocardial angiogenesis and can mediate cardiomyocyte hypertrophy. In addition, STAT3 is involved in protection of the heart from pregnancy-induced oxidative stress by inhibition of the reactive oxygen species by upregulation of mangenase superoxide dismutase (MnSOD). Recently, an article was published in which STAT3 knockout (KO) mice showed normal pregnancy-induced vessel growth and hypertrophy but displayed increased apoptosis, loss of myocardial capillaries, fibrosis and dilatation in postpartum period [20]. The STAT3 pathway is activated by prolactin, which is produced by the pituitary gland. Prolactin exists in a fulllength $23-\mathrm{kDa}$ form, which can be cleaved by the protease cathepsin $\mathrm{D}$ into an antioangiogenic and proapoptotic $16-\mathrm{kDa}$ fragment (Fig. 1). A recent investigation demonstrated the unfavourable influence of $16-\mathrm{kDa}$ prolactin. Systemic infusion of 23-kDa prolactin in wild-type mice and STAT3 KO mice had no adverse effects on the heart. However, expression of the 16-kDa prolactin in the heart destroyed the cardiac microvasculature, reduced the cardiac function (in vivo) and decreased the cardiomyocyte metabolism, even in absence of pregnancy. A recent study showed that bromocriptine inhibits $23-\mathrm{kDa}$ prolactin. Thereby, it eliminates the substrate for the generation of $16-\mathrm{kDa}$ prolactin and prevents PPCM in STAT3-KO mice [20].

\section{Diagnosis and Clinical Presentation}

Patients with PPCM present with typical signs and symptoms of left ventricular failure. Owing to the similarity with normally occurring pregnancy-associated complaints, the symptoms of PPCM are often misinterpreted, which prolongs the time lag for diagnosis and the correct treatment. The delay in reaching the correct diagnosis has been estimated from weeks to months in approximately $30 \%$ of the cases [21]. Normal pregnancies are often associated with non-specific fatigue, pedal oedema, inability to lay supine and decreased exercise capacity. Symptoms that should raise suspicion of cardiac failure include nocturnal cough, orthopnoea, paroxysmal nocturnal 
Fig. 1 Development of PPCM. In the absence of cardiomyocyte STAT3 activity, the amount of MnSOD will decrease. This leads to an increase of oxidative stress and the release of cathep$\sin \mathrm{D}$, which processes $23-\mathrm{kDa}$ prolactin into the $16-\mathrm{kDa}$ form. The 16-kDa prolactin induces endothelial cell apoptosis, capillary dissociation, vasoconstriction and impairs cardiomyocyte metabolism, thereby promoting PPCM. Accordingly, bromocriptine, a pharmacological inhibitor of prolactin release, prevents PPCM by inhibiting 23-kDa prolactin. $c D$ cathepsin $\mathrm{D}, R O S$ reactive oxygen species, $M n S O D$ manganese superoxide dismutase

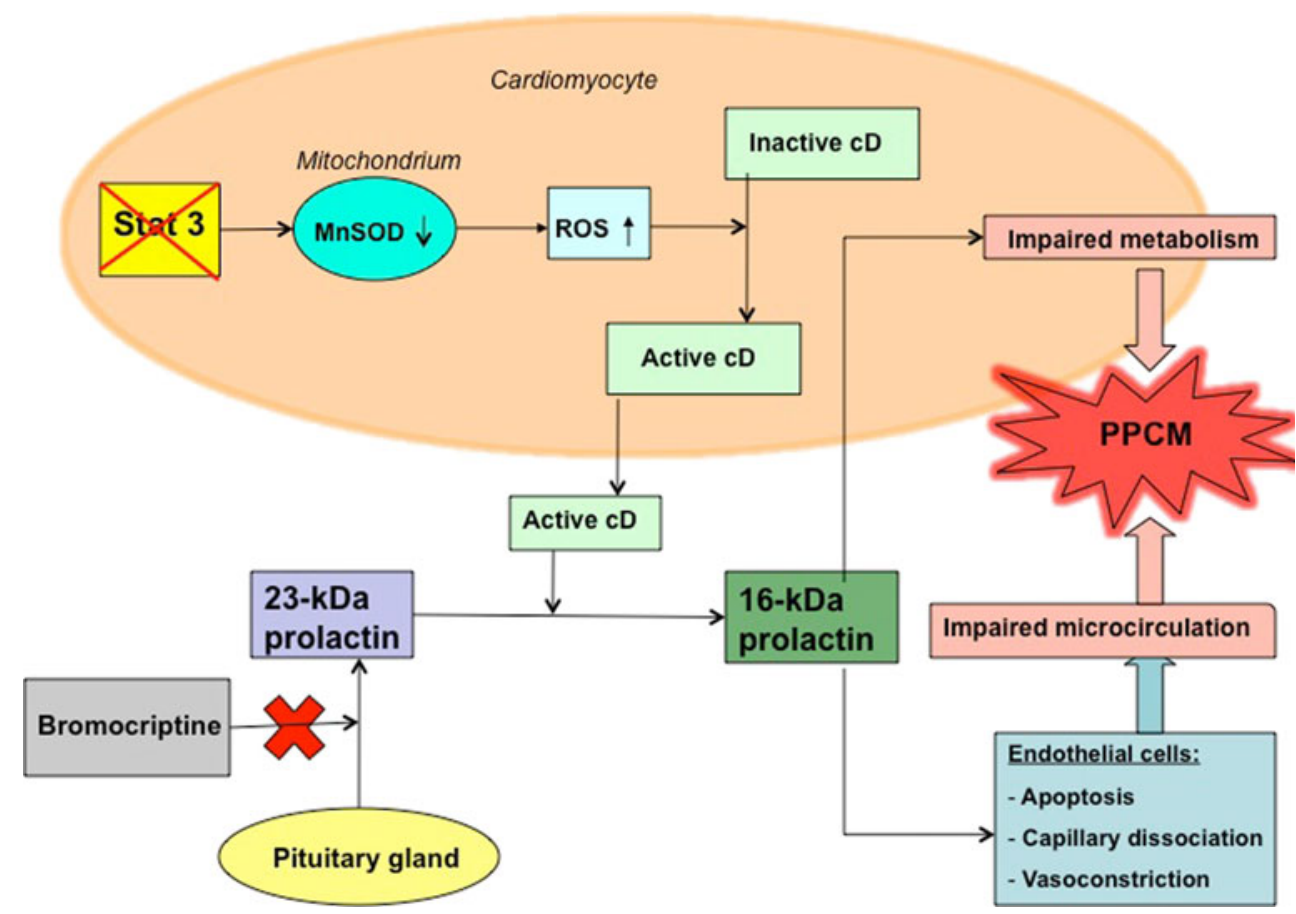

dyspnoea, new murmurs of atrioventricular valvular regurgitation and haemoptysis [6].

\section{Physical Examination}

Physical examination of pregnant women often reveals peripheral oedema. Most pregnant women have audible physiological systolic murmurs, created by augmented blood flow. Patients with PPCM may display signs of cardiac enlargement, a third heart sound (S3) and murmurs of mitral and tricuspid regurgitations.

\section{Differential Diagnosis}

The differential diagnosis of PPCM includes anaemia, thyroid disorders, accelerated hypertension, diastolic dysfunction, myocardial infarction, sepsis, severe preeclampsia, amniotic fluid embolism and pulmonary embolism [22]. Pulmonary embolism is an important differential diagnosis as it is the leading cause of maternal death. In both cases, the progression of symptoms is rapid and can lead to death in a short time after the initial presentation. However, management differs substantially.

\section{Diagnostic Procedures}

When a diagnosis of PPCM is suspected, every effort must be made to exclude pre-existing cardiac disease or other underlying disorders. A detailed history is the most important tool to distinguish a pre-existent cardiomyopathy with PPCM. Establishing a specific cause for left ventricular failure excludes the diagnosis of PPCM. An electrocardiogram and an echocardiogram should be performed in all patients suspected of PPCM. In addition, a MRI can be used.

\section{Electrocardiogram}

An ECG may act as an initial screening tool but is not suitable for an unambiguous diagnosis of PPCM. Possible ECG findings include sinus tachycardia and non-specific ST and $\mathrm{T}$ wave abnormalities. In addition, voltage abnormalities, such as low voltage or oppositely left ventricular hypertrophy, can be seen. Q waves are occasionally present in the anterior leads. Finally, P-R and QRS intervals may be prolonged. However, it is important to state that a normal ECG does not exclude PPCM [6].

\section{Echocardiogram}

An echocardiogram appears to be extremely valuable in diagnosing PPCM. Echocardiography can give vital information concerning left and right ventricular systolic and diastolic function. In normal pregnancy, despite increased left ventricular volume and dimension, most echocardiographic characteristics of left ventricular function, such as ejection fraction, fractional shortening and contractility, are similar to nonpregnant women [23]. In contrast, an 
echocardiogram in PPCM usually reveals enlargement of all four chambers, with marked reduction in left ventricular systolic function. Small to moderate pericardial effusion and mitral and tricuspid regurgitation (secondary to ventricular dilatation) may also be evident $[6,9]$.

\section{MRI}

Cardiac MRI is safe, free of radiation, and offers a global view of the myocardium. Cine MRI gives a reproducible and reliable evaluation of the global and segmental myocardial contraction. Recently, Mouquet et al. investigated eight patients who were diagnosed with PPCM. The patients did not exhibit a specific cardiac MRI pattern and importantly no myocardial late enhancement, as is often seen in idiopathic dilated cardiomyopathy. Therefore, myocardial fibrosis was thought not to play a major limiting role in cardiac recovery after PPCM [24]. However, MRI with delayed enhancement can detect large areas with cardiac fibrosis but is not sensitive enough to detect interstitial fibrosis. In addition, due to the small sample size, incorrect nomenclature and the lack of standardisation of cardiac magnetic resonance protocols, a large prospective and systematic study of the prognostic value of fibrosis in PPCM is necessary.

\section{Serum Markers}

As in most forms of heart failure, case reports have documented that serum levels of troponin $\mathrm{T}$ and creatine kinase are hardly increased in patients with PPCM. BNP levels in pregnancy are approximately twofold increased and do not significantly fluctuate during pregnancy. The rise in BNP level is seen as early as the first trimester and may persist up to the 24th week after delivery [25]. INF- $\gamma$ (a marker of inflammation), prolactin and oxidised LDL (marker for oxidative stress) are significantly elevated in PPCM patients in comparison with healthy pregnant women [26]. Reversal of these parameters predicted a better outcome. These results suggest that a specific prognostic biomarker profile may exist in PPCM patients, which might be used for diagnosis and risk stratification in the future.

\section{Endomyocardial Biopsy}

The role of routine endomyocardial biopsy in the diagnosis of PPCM is controversial and not standard practice. There is an inherent risk of perforation and cardiac tamponade [27].

\section{Treatment}

Treatment of PPCM is rather similar to other types of congestive heart failure. However, concerns regarding the adverse effects of this therapy for the mother, foetus or breastfeeding infant may complicate the management of patients with PPCM. Murali et al. reviewed that conventional pharmacological therapy with diuretics, sodium restriction, $\beta$-blockers, digoxin and afterload reduction forms the cornerstone of therapy. Angiotensin-converting enzyme (ACE) inhibitors are absolutely contraindicated during the second and third trimester of the pregnancy because of the risk of kidney disturbances of the foetus and oligohydramnion. Use of ACE inhibitors in breastfeeding patients is also not recommended [3].

\section{Antithrombotic Therapy}

Pregnancy is accompanied by physiological changes in coagulation that may have evolved to protect women from the bleeding risk of miscarriage and birth. Many of the clotting factors increase and anticoagulation factors decrease. This augmented coagulation and decreased fibrinolysis, together with the increased risk for ventricular thrombi as a result of depressed cardiac function, emphasises that PPCM patients have an increased risk for thromboembolic events. Warfarin is contraindicated in pregnancy. It crosses the placenta and is associated with teratogenic effects in the foetus. However, most of these effects occur particularly between the 6th and 12th weeks of gestation. As a consequence, warfarin can be used safely during the third trimester, the moment most PPCM patients are being diagnosed. However, warfarin is a long-acting anticoagulant; therefore, its effect could lead to haemorrhagic complications in the newborn, or in pregnant patients with preterm delivery or in need of emergent caesarean section. In comparison, the short half-life of heparin allows it to be discontinued prior to delivery to prevent maternal haemorrhage. Heparin is a large molecule, so it does not cross the placenta and does not cause teratogenic effects. Hence, subcutaneous heparin is the drug of choice for the pregnant PPCM patient. For the postpartum patient, either heparin or warfarin may be used safely. Neither drug is secreted into breast milk; therefore, the infant is not at risk of an anticoagulated state [3].

\section{Arrhythmias}

Ventricular arrhythmias should be managed aggressively. Beta-blocking agents are effective in preventing ventricular arrhythmias. In addition, the use of class III antiarrhythmic drugs, such as sotalol and amiodarone, can also be 
considered. However, these drugs have many side effects during chronic use, and these should be carefully weighed before they are prescribed. Amiodarone may be toxic for mother and baby, leading to hyperthyroidism and retarded growth, with the risk of perinatal death. Patients with symptomatic ventricular arrhythmias may be candidates for defibrillator implantation (ICD). However, it seems better to await the recovery of cardiac function because the disease process is potentially reversible [3].

\section{Bromocriptine}

A recent small study investigated the role of bromocriptine in women with a subsequent pregnancy. A total of 12 pregnant women who suffered from PPCM in a previous pregnancy were divided into two groups. Six patients were given bromocriptine in addition to standard heart failure therapy. All of these patients had an uneventful post-pregnancy follow-up. In contrast, all patients in the group who only received standard heart failure therapy suffered from recurrence of PPCM. Furthermore, three patients in the nonbromocriptine group died [20]. In addition, case reports confirm recovery of the heart in patients with PPCM who were treated with bromocriptine in addition to heart failure therapy. Controlled randomised studies are awaited to provide evidence for this promising novel therapy. However, there are case reports on myocardial infarction due to thromboembolic events in postpartum patients, which occurred in association with taking bromocriptine [28]. Therefore, it is recommended that bromocriptine should only be applied in combination with low-dose heparin.

\section{Mechanical Circulatory Support and Heart Transplantation}

Aggressive life-support measures, such as ventricular assist devices, should be used when more conservative therapy is not adequate to sustain life, until cardiac recovery occurs $[2,3]$. Heart transplantation should only be considered if the heart does not recover, and this may take considerable amount of time.

\section{Prognosis}

A number of studies have evaluated the outcome of women with PPCM and vary with regard to maternal prognosis. In approximately two thirds of the PPCM patients, improvement of the left ventricular function was seen on echocardiogram, and $45 \%$ of the patients returned to a normal ejection fraction $(\mathrm{EF}>50 \%)[10,29]$. In contrast, $10 \%$ of the patients required heart transplantation [29]. Recent studies by Mielniczuk et al. and Brar et al. revealed mortality rates of $2.1 \%$ and $3.3 \%$ $[30,31]$. This is substantially lower than the $32 \%$ 6-month mortality reported by Sliwa et al. in their series from South Africa [32]. The variability in mortality may be related to improvement in medical therapy for heart failure and the more liberal use of mechanical circulatory support. Despite these encouraging statistics, a small subset of women remains whose illness follows a rapid and irreversible course that ultimately leads to persistent left ventricular dysfunction, chronic heart failure, heart transplantation or premature death. Even among patients whose resting left ventricular systolic function normalises, the contractile reserve of the left ventricle, when challenged with dobutamine, often remains diminished [33].

Factors that indicate a poor prognosis in PPCM include a lower left ventricular ejection fraction at 6 months after delivery, larger left ventricular end-diastolic dimension, age over 30 years old, African American descent and multiparity [3].

\section{Subsequent Pregnancies}

Subsequent pregnancies in patients with PPCM are associated with a significant effect on both maternal and foetal outcome. Therefore, it is very important to inform all PPCM patients of the high risk of recurrence of PPCM during or after a subsequent pregnancy, even if their heart function seems to have recovered completely.

The incidence of complications is higher in women with persistent left ventricular dysfunction. In addition, there is an increased risk of foetal prematurity and foetal loss. Although the likelihood of maternal death seems to be very low in women with recovered left ventricular function, a persistent reduction in left ventricular ejection fraction and symptomatic heart failure may occur during subsequent pregnancies. Elkayam et al. investigated PPCM patients with a subsequent pregnancy. Of the patients whose heart function seemed to have recovered, $21 \%$ developed symptoms of heart failure in the subsequent pregnancy versus $44 \%$ of patients with impaired systolic function [34].

Recurrent symptomatic heart failure in patients with a history of PPCM and recovered left ventricular function has been attributed to a physiological increase in blood volume, stroke volume and heart rate during pregnancy. These haemodynamic changes are expected to cause symptomatic deterioration in patients with persistent subclinical myocardial dysfunction, which may exist even in patients with apparent recovery of left ventricular function. However, a significant depression of left ventricular function associated with subsequent pregnancy suggests that worsening of symptoms may also be due to the underlying idiopathic 
process responsible for the development of the cardiomyopathy in the previous pregnancy [33].

If a patient with a previous PPCM becomes pregnant again, $\beta$-blockers can be used safely. Beta-1-selective blockers are preferable, since these agents are less likely to interfere with $\beta$-2-mediated uterine relaxation and peripheral vasodilatation. After the first trimester of a subsequent pregnancy, ACE inhibitors should not be used [3]. As already mentioned, there might be a role for bromocriptine in patients with PPCM and subsequent pregnancies, but this awaits substantiation by larger trials [20].

\section{Conclusion}

Peripartum cardiomyopathy is a rare form of dilated cardiomyopathy with left ventricular systolic dysfunction that affects women in late pregnancy or in the puerperium. The incidence of PPCM is unknown. Basic research suggests a role for bromocriptine in the prevention of this potentially fatal disease, but randomised clinical trials are lacking. We therefore have the intention to start a national database for PPCM patients. This will be useful for gathering epidemiological information to determine the magnitude of the problem. In addition, the database will serve as a depository for tissue and blood samples to answer fundamental questions on the pathogenesis. Furthermore, it will allow us to initiate randomised trials to investigate the optimal treatment for this disease.

Open Access This article is distributed under the terms of the Creative Commons Attribution Noncommercial License which permits any noncommercial use, distribution, and reproduction in any medium, provided the original author(s) and source are credited.

\section{References}

1. Bosch MGE, Santema JG, van der Voort PHJ, et al. A serious complication in the puerperium: peripartum cardiomyopathy. Neth Heart J. 2008;16:415-8.

2. Oosterom L, de Jonge N, Kirkels J, et al. Left ventricular assist device as a bridge to recovery in a young woman admitted with peripartum cardiomyopathy. Neth Heart J. 2008;16:426-8.

3. Murali S, Marie M, Baldisseri M. Peripartum cardiomyopathy. Crit Care Med. 2005;33:340-6.

4. Pearson G, Veille J, Rahimtoola S, et al. Peripartum cardiomyopathy: National Heart, Lung and Blood Institute and office of rare diseases (National Institute of Health) workshop recommendations and review. JAMA. 2000;283:1183-188.

5. Forssell G, Laska J, Olofsson C, et al. Peripartum cardiomyopathy: three cases. J Intern Med. 1994;235:493-6.

6. Elkyam U, Akhter MW, Singh H, et al. Pregnancy-associated cardiomyopathy. Clinical characteristics and a comparison between early and late presentation. Circulation. 2005;111:2050-5.
7. Sliwa K, Fett J, Elkayam U. Peripartum cardiomyopathy. Lancet. 2006;368:687-93.

8. Mayosi BM. Contempory trends in the epidemiology and management of cardiomyopathy and pericarditis in sub-Saharan Africa. Heart. 2007;93:1176-83.

9. Fett JD, Christie LG, Carraway RD, et al. Five-year prospective study of the incidence and prognosis of peripartum cardiomyopathy at a single institution. Mayo Clin Proc. 2005;80:1602-6.

10. Bultmann BD, Klingel K, Nabauer M, et al. High prevalence of viral genomes and inflammation in peripartum cardiomyopathy. Am J Obstet Gynecol. 2005;193:363-5.

11. Felker GM, Jaeger CJ, Klodas E, et al. Myocarditis and long-term survival in peripartum cardiomyopathy. Am Heart J. 2000;140:785-91.

12. Rizeq MN, Rickenberger PR, Fowler MB, et al. Incidence of myocarditis in peripartum cardiomyopathy. Am J Cardiol. 1994;74:474-7.

13. Nelson J. Pregnancy, persistent microchemerism and autoimmune disease. J Am Med Women Assoc. 1998;53:31-6.

14. Ansari A, Neckelman N, Wang Y, et al. Immunologic dialogue between cardiac myocytes, endothelial cells and mononuclear cells. Clin Immunol Immunopathol. 1993;68:208-14.

15. Sliwa K, Skudicky D, Bergman A, et al. Peripartum cardiomyopathy: analysis of clinical outcome, left ventricular function, plasma levels of cytokines and Fas/APO-1. J Am Coll Cardiol. 2000;35:701-5.

16. Gonzalez AM, Osorio JC, Manlhiot C, et al. Hypertrophy signaling during peripartum cardiac remodeling. Am J Physiol Heart Circ Physiol. 2007;293:3008-13.

17. Hayakawa Y, Chandra M, Miao W, et al. Inhibition of cardiac myocyte apoptosis improves cardiac function and abolishes mortality in the peripartum cardiomyopathy of $\mathrm{G} \alpha \mathrm{q}$ transgenic mice. Circulation. 2008;117:396-404.

18. Meyer GP, Labidi S, Podewski E, et al. Bromocriptine treatment associated with recovery from peripartum cardiomyopathy in siblings: two case reports. J Med Case Rep. 2010;4:80.

19. Spaendonck-Zwarts KY, van Tintelen JP, van Veldhuisen DJ, et al. Peripartum cardiomyopathy as a part of familial dilated cardiomyopathy. Circulation. 2010;121:2169-75.

20. Hilfiker-Kleiner D, Kaminski K, Podewski E, et al. A cathepsin D-cleaved $16 \mathrm{kDa}$ form of prolactin mediates postpartum cardiomyopathy. Cell. 2007;128:589-600.

21. Selle T, Renger I, Labidi S, et al. Reviewing peripartum cardiomyopathy: current state of knowledge. Future Med. 2009;5:175-89.

22. Ray P, Murphy J, Shutt L, et al. Recognition and management of maternal cardiac disease in pregnancy. Br J Anaesth. 2004;93:428-39.

23. Geva T, Mauer MB, Striker L, et al. Effects of physiologic load of pregnancy on left ventricular contractility and remodeling. Am Heart J. 1997;133:53-9.

24. Mouquet F, Lions C, de Groote P, et al. Characterisation of peripartum cardiomyopathy by cardiac magnetic resonance imaging. Eur Radiol. 2008;18:2765-9.

25. Hameed A, Chan K, Ghamsary M, et al. Longitudinal changes in the B-type natriuretic peptide levels in the normal pregnancy and postpartum. Clin Cardiol. 2009;32:60-2.

26. Forster O, Hilfiker-Kleiner D, Ansari AA, et al. Reversal of IFN$\gamma$, oxLDL and prolactin serum levels correlate with clinical improvement in patients with peripartum cardiomyopathy. Eur J Heart Fail. 2008;10:861-8.

27. Baughman K. Peripartum cardiomyopathy. Curr Treat Options Cardiovasc Med. 2001;3:469-80.

28. Hopp L, Haider B, Iffy L. Myocardial infarction postpartum in patients taking bromocriptine for the prevention of breast engorgement. Int J Cardiol. 1996;57:227-32.

29. Amos AM, Jaber W, Russell SD. Improved outcome in peripartum cardiomyopathy with contemporary. Am Heart J. 2006;152:509-13. 
30. Mielniczuk LM, Williams K, Dadvis DR, et al. Frequency of peripartum cardiomyopathy. Am J Cardiol. 2006;97:1765-8.

31. Brar SS, Khan SS, Sandhu GK, et al. Incidence, mortality and racial differences in peripartum cardiomyopathy. Am J Cardiol. 2007;100:302-4.

32. Sliwa K, Skundicky D, Bergemann A, et al. Peripartum cardiomyopathy: analysis of clinical outcome, left ventricular function, plasma levels of cytokines and Fas/APO-1. J Am Coll Cardiol. 2000;35:701-5.

33. Lampert MB, Weinert L, Hibbard J, et al. Contractile reserve in patients with peripartum cardiomyopathy and recovered left ventricular function. Am J Obstet Gynecol. 1997;176:189-95.

34. Elkayam U. Pregnant after peripartum cardiomyopathy: to be or not to be? Eur Heart J. 2002;23:753-6. 